\title{
A NORMAL SPACE $X$ FOR WHICH $X \times I$ IS NOT NORMAL
}

\author{
BY MARY ELLEN RUDIN
}

Communicated by E. A. Michael, September 28, 1970

In [1] C. Dowker gave a number of interesting characterizations of normal Hausdorff spaces whose cartesian product with the closed unit interval is not normal. Thus, such a space is often called a Dowker space; a Dowker space $X$ will be described below. It was previously known only that the existence of a Dowker space is consistent with the usual axioms of set theory [2], [3]. The proof that $X$ is a Dowker space uses no set theoretic assumptions beyond the axiom of choice.

We use the convention that an ordinal $\lambda$ is the set of all ordinals less than $\lambda$. An ordinal $\gamma$ is said to be cofinal with $\lambda$ if there is a subset $\Gamma$ of $\lambda$ order isomorphic with $\gamma$ such that $\alpha \in \lambda$ implies $\alpha \leqq \beta$ for some $\beta \in \Gamma$; let $\operatorname{cf}(\lambda)$ be the smallest ordinal cofinal with $\lambda$.

Define $F=\left\{f: \omega_{0} \rightarrow \omega_{\omega} \mid \forall n \in \omega_{0}, f(n) \leqq \omega_{n}+1\right\}$.

Define $X=\left\{f \in F \mid \exists k \in \omega_{0}\right.$ such that $\left.\forall n \in \omega_{0}, \omega_{0}<\operatorname{cf}(f(n))<\omega_{k}\right\}$.

For $f$ and $g$ in $F$, define $U_{f g}=\left\{h \in X \mid \forall n \in \omega_{0}, f(n)<h(n) \leqq g(n)\right\}$. Then topologize $X$ by using the set of all $U_{f g}$ for $f$ and $g$ in $F$ as a basis for the topology. The resulting space is a collectionwise normal Dowker space.

\section{REFERENCES}

1. C. H. Dowker, On countably paracompact spaces, Canad. J. Math. 3 (1951), 219-224. MR 13, 264.

2. M. E. Rudin, Countable paracompactness and Souslin's problem, Canad. J. Math. 7 (1953), 543-547. MR 17, 391.

3. S. Tennenbaum, Souslin's problem, Proc. Nat. Acad. Sci. U. S. A. 59 (1968), 60-63. MR $37 \# 55$.

University of Wisconsin, Madison, Wisconsin 53706

AMS 1969 subject classifications. Primary 5420, 0415, 0430.

Key words and phrases. Dowker space, cartesian product, normal, binormal, collectionwise normal, countably paracompact. 